\title{
Asymmetry Map of Human Brain in Young Adults
}

Maryam Malekzadeh $\mathrm{MSc}^{1}$, Alireza Kashani MD, $\mathrm{PhD}^{1^{*}}$

1. Shahid Beheshti University, Tehran 1983969411, Iran

*Author to whom correspondence should be addressed: a.kashani@medneuron.com

\begin{abstract}
Although, asymmetry is a central organizational aspect of human brain, it has not been clearly described yet. Here, we have studied structural brain asymmetry in 1113 young adults using data obtained from Human Connectome Project. A significant rightward asymmetry in mean global cerebral cortical thickness, surface area and gray matter volume as well as volumes of cerebral white matter, cerebellar cortex and white matter, hippocampus, putamen, caudate nucleus, nucleus accumbens and amygdala was observed. Thalamus showed a leftward asymmetry. Regionally, most cerebral cortical regions show a significant rightward asymmetry in thickness. However, cortical surface area and gray matter volume are more evenly distributed between two hemispheres with almost half of the regions showing a leftward asymmetry. In addition, a strong correlation between cortical surface area and gray matter volume as well as their asymmetry indices was noted which results in concordant asymmetry patterns between cortical surface area and gray matter volume in most cortical regions.
\end{abstract}

\section{Key words}

Asymmetry, Human Brain, Young Adult, Gender, Human Connectome Project 


\section{INTRODUCTION}

Human brain is a lateralized structure (1). Several lines of evidence suggest that this feature is centrally involved in numerous tasks including cognitive and affective processes in physiological as well as pathological states (2-7). However, despite over a century of research, this important hallmark has not been clearly understood yet. To date, different and frequently conflicting reports about how human brain is lateralized and affected by variables like gender and age have been published (8-14). Variability in methods and populations, subtle and varying nature of asymmetry with age as well as vast inherent individual variability are considered some of the reasons for previous inconsistent reports (11, 13, 15-18). Young Adult Human Connectome Project (HCP) has applied standardized parcellation and imaging methods to study 300 families of 22-35 years old twin and non-twin siblings which are diverse enough to reflects ethnic diversity of the United States (19). This provides an excellent opportunity to study brain asymmetry in young adults and how is affected by specific variables like gender. Furthermore, HCP data set allows us to study asymmetry in different cortical and subcortical structures in same group of participants since ever growing data indicate that they actively participate in cardinal brain functions (20-22). In this study, we have studied asymmetry patterns in three measures of the cerebral cortex: thickness, surface area, gray matter volume, as well as cerebral white matter, cerebellar cortex and white matter, hippocampus, pallidum, putamen, caudate nucleus, nucleus accumbens, amygdala and thalamus.

\section{MATERIALS AND METHODS}

All analyses were performed on datasets downloaded from the Young Adult HCP database (19). The process of acquisition and processing of the MRI images are 
explained here (23). The structural data of 1113 participants, 606 female and 507 male, who were between 22-35 years old was analyzed. Statistical analyses were performed using SPSS software ver. 24.0 (IBM, Armonk, NY, USA). Asymmetry Index (AI) of thickness, surface area and gray matter volume of each cortical region as well as volumes of cerebral white matter, hippocampus, pallidum, putamen, caudate nucleus, nucleus accumbens, amygdala and thalamus was calculated for each subject according to the formula of $2 \times$ (left-right)/(left+right). Positive and negative Als indicate leftward or rightward asymmetry respectively. A single group $t$-test was carried out for comparison of Al means. A significance level of $P<0.0011$ ( $0.05 / 44$ brain regions) was assumed due to Bonferroni corrections to prevent inflated error rates. Effect size was expressed as Cohen's-d (d). A Multivariate Analysis of Variance (MANOVA) test was conducted to evaluate effect of gender as between-subject factor and age as a covariate on Als of studied structures. Results were analyzed with region specific post hoc tests. Coefficient of Variation (CV) was calculated according to the formula: $\mathrm{CV}=$ standard deviation/mean to evaluate variability of metrics in the cerebral cortex. Pearson correlation coefficient $(R)$ was calculated to study relationship between them. For correlation analysis statistical significance threshold was set at $P<0.05$.

\section{RESULTS}

\section{Cerebral Cortex}

A significant rightward asymmetry is observed in mean global cortical thickness, surface area as well as mean global cortical gray matter and cerebral white matter volumes. (Table.1 and Fig.1). However, at regional level differential asymmetry patterns were observed as follows. 
1. Thickness. $85 \%(29 / 34)$ of cerebral cortical regions show a significant degree of asymmetry in thickness with $76 \%$ (26/34) presenting a rightward asymmetry. Insula, caudal anterior and posterior cingulate cortices show a significant leftward asymmetry. Parahippocampal and precentral gyri as well as rostral anterior cingulate, pericalcarine and cuneus cortices display a non-significant difference. All other regions show a significant rightward asymmetry in thickness (Table.2 and Fig.2).

2. Area. $94 \%(32 / 34)$ of the cerebral cortical regions present a significant asymmetry in surface area. However, distribution of regions between two hemispheres is more even relative to cortical thickness. $41 \%$ (14/34) show a rightward and 53\% (18/34) present a leftward asymmetry. Regions with rightward asymmetry are widely distributed on all cerebral surfaces including frontal pole, pars orbitalis, pars triangularis, insula, middle temporal gyrus and inferior parietal, pericalcarine, caudal anterior cingulate cortices. Regions with leftward asymmetry show a tendency to peri-Sylvian areas and include superior temporal and supramarginal gyri, temporal pole, pars opercularis as well as transverse temporal, entorhinal and rostral anterior cingulate cortices (Table.3 and Fig.3).

3. Gray matter volume. $88 \%(30 / 34)$ of cerebral cortical regions show a significant asymmetry in their gray matter volumes. 41\% (14/34) display a rightward and 47\% (16/34) a leftward asymmetry. Regions with leftward asymmetry indices include transverse temporal, rostral anterior cingulate, pars opercularis, caudal anterior cingulate and entorhinal cortices. On the other hand, middle temporal gyrus, pars triangularis, banks of superior temporal sulcus, inferior parietal cortex, pars orbitalis and frontal pole regions present a rightward asymmetry (Table.4 and Fig.4).

\section{Cerebellum, Hippocampus and Subcortical Structures}


Mean of volumes of the cerebellar cortex and white matter, hippocampus, pallidum, putamen, caudate nucleus, nucleus accumbens and amygdala show a significant rightward asymmetry. Thalamus present a significant leftward asymmetry (Table.5 and Fig.5).

\section{Variation of the Cerebral Cortical Metrics}

Cerebral cortical surface area $(C V=14)$ and gray matter volume $(C V=14)$ display more global variability than thickness (CV=6) (Fig.6A).

\section{Correlation between the Cerebral Cortical Metrics}

We observed a tendency towards a negative correlation between cortical thickness and surface area across cortical regions $\left(\mathrm{R}^{2}=0.04, P=0.24\right)$ as well as the corresponding Als $\left(\mathrm{R}^{2}=0.1, P=0.08\right)$ which do not reach the statistical significance. Furthermore, thickness and the gray matter volume do not show a significant correlation $(\mathrm{R} 2=0.032, P=0.31)$, neither their corresponding Als (R2=0.003, $P=0.76406)$. However, surface area and gray matter volume $\left(\mathrm{R}^{2}=0.96, P<0000001\right)$ as well as their corresponding Als $\left(\mathrm{R}^{2}=0.6, P<\right.$ 0000001) present a strong and significant correlation together (Fig.6 and 7).

\section{Gender}

MANOVA revealed a significant effect of gender on Als of the studied structures [F (113, 998) $1.9 ; P<0$. 0.0000005, partial $\eta 2=0.18]$. However, even though, a number of nominally significant regions with exaggerated asymmetry patterns between genders was noted in post-hoc analysis, none survived Bonferroni correction (Table.6).

\section{Age}

Age does not show a significant effect on Als of studied structures $[F(113,998) 1 ; P=$ 0.35 , partial $\eta 2=0.11]$. 


\section{DISCUSSION}

To best of our knowledge, this is, by far, the largest study of asymmetry in healthy young adults which have been analyzed by standardized parcellation and imaging methods. Previous reports have either studied smaller groups which would not provide necessary statistical power or analyzed the data which have been obtained through heterogeneous methods. Moreover, this is the first study which has studied asymmetry in three metrics of the cerebral cortex as well as cerebral white matter, cerebellar cortex and white matter, hippocampus and six subcortical structures in the same group of individuals. A major finding of this study is the almost ubiquitous rightward asymmetry in mean of gray and white matter volumes in the studied structures. Volumes of the cerebral as well as cerebellar cortex and white matter, hippocampus and all subcortical structures expect thalamus show a significant rightward asymmetry. In addition, mean global cerebral cortical thickness and surface area present a rightward asymmetry as well (Table.1 and 5), (Fig.1 and 5). The findings are in agreement with a previous study describing a rightward asymmetry in the cortical gray matter as well as cerebral white matter volumes (24). In addition, although, only a few studies has addressed hemispheric asymmetry in cortical surface area, thickness and gray matter volume in the same group of participants, a global rightward asymmetry in all these metrics has been reported before (25). Moreover, rightward asymmetry of the cerebral gray matter volume, and cortical surface area has been described in other studies $(13,26)$. Nevertheless, conflicting asymmetry patterns have been also reported $(12,13,27,28)$. Despite the global rightward asymmetry of cortical metrics, differential patterns at regional level were observed. Although, most cortical regions show a rightward asymmetry in thickness (Table.2 and Fig.2), surface 
area presents a more even distribution between two hemispheres with $53 \%$ of regions showing a significant leftward asymmetry (Table.3 and Fig.3). It is notable that in the lateral surface of the cerebral cortex, asymmetry in surface area shows a relationship with the distance to Sylvian fissure leading to a leftward asymmetry in peri-Sylvian regions which has been frequently reported before $(13,25,29-31)$. For example, both caudal middle and inferior frontal (pars opercularis) gyri display a leftward asymmetry in surface area. However, rostral middle and inferior frontal (pars orbitalis and pars triangularis) gyri show a rightward asymmetry. A similar pattern is observed in parietal cortex. Postcentral and supramarginal gyri show a leftward asymmetry in surface area. However, inferior and superior parietal cortices show a rightward asymmetry. In medial surface of cerebrum an anterior-left, posterior-right pattern of asymmetry in surface area is observed. Superior frontal as wells as rostral anterior cingulate regions show a leftward asymmetry. However, precuneous, cuneous, caudal anterior and posterior cingulate cortices show a rightward asymmetry. Between structural variables of cortical gray matter volume (32), surface area shows more global variability $(C V=14)$ compared with thickness $(C V=6)($ Fig.6A), which is in agreement with previous reports $(29,33)$. This is probably why global variability $(\mathrm{CV}=14 \%)$ as well as the volume of cortical gray matter often follows cortical surface area $\left(\mathrm{R}^{2}=0.96, P<0000001\right)$ (Fig.6). Concordantly, the asymmetry patterns of the cortical gray matter volume mostly pursue surface area (Fig.7A). This includes leftward asymmetry of cortical gray matter volume in peri-Sylvian regions including transvers temporal cortex, superior temporal gyrus, temporale pole, pars opercularis and supramarginal gyrus which is in agreement with previous reports (Table.4 and Fig.4) (9, 10, 26, 34). Regions with concordant rightward asymmetry between surface area and gray matter volume include 
middle temporal gyrus, pars orbitalis, pars triangularis, insula, inferior parietal, cuneous, precuneous and posterior cingulate cortices which are mostly in line with a previous study (30). Regions with opposite asymmetry pattern between surface area and gray matter volume are not numerous and include banks of superior temporal sulcus, caudal rostral cingulate and medial orbitofrontal gyri where gray matter volume follow asymmetry pattern of the cortical thickness (Table.2 , 4 and Fig.2, 4). Consistently, unlike a previous report (25), we noticed a strong global relationship between Als of the cortical surface area and gray matter $(\mathrm{R}=0.6, P<0000001)$ compared with a non-significant relationship between Als of the cortical thickness and gray matter $(\mathrm{R} 2=0.003, P=0.76406)$ which is in line with a previous report (33). Fig. 7B). This supports the notion of independent character of cortical thickness and surface area as neuroanatomical unites $(30,33,35)$. A good example of how variation in surface area influences asymmetry of the gray matter volume is Broca's area. Although, both parts of Broca's area, pars triangularis and pars opercularis, present a rightward asymmetry in thickness, the gray matter volume follows asymmetry pattern of surface area, i.e. rightward in pars triangularis and leftward in pars opercularis. In fact, difference in surface area asymmetry places the gray matter volume asymmetry of pars triangularis $(d=-1.2)$ and pars opercularis $(d=1.1)$ among strongest opposite asymmetries in whole brain. Interestingly, a similar pattern is observed in another language related region, supramarginal gyrus i.e. a rightward asymmetry in thickness and leftward asymmetry in surface area and gray matter volume. Although, many of the asymmetry patterns in cortical metrics described here, has been reported in different studies $(9,13,25,26,28-30)$, it is the first time, in our knowledge, that above mentioned relationship between cortical metrics in determining asymmetry patterns 
across cerebral cortex has been described. The results of this study in regional cortical asymmetry are similar to a previous report (30). However, probably lower number of cases and lack of necessary statistical power in addition of strict correcting for multiple comparisons by Bonferroni procedure has led to many statistically insignificant results in above mentioned study. In addition, we could replicate many of the asymmetry patterns reported by other studies $(12,29,31)$, even though, inconsistencies exist which detailed discussion about it is beyond the scope of this paper. However, we have to emphasize that a part of the inconsistencies could be due to the age of participants as reports suggest it affects asymmetry patterns $(17,18)$. On the other hand, although, our results show similarities with another large data analysis (13), especially on asymmetries in cortical surface area, we could not replicate reported leftward hemispheric asymmetry as well as many regional asymmetries in cortical thickness. Important heterogeneity in asymmetry results between analyzed data sets which has been acknowledged by the authors could be one of the reasons of this discrepancy (13).

\section{Cerebellum, Hippocampus and Subcortical Structures}

Although, fewer studies on the asymmetry of subcortical structures are available, discrepancies prevail here as well. Apart from putamen and pallidum, the observed rightward asymmetry in hippocampus, amygdala, caudate nucleus and nucleus accumbens as well as leftward asymmetry in thalamus are in agreement with the largest met-analysis published on asymmetry of subcortical structures (11) as well as other reports $(27,36)$. The reason of the reported leftward asymmetry in putamen and pallidum in above mentioned studies can be a more rapid decline in volume of right putamen and pallidum and a leftward shift in the asymmetry with age which has been reported before 
$(11,37)$. The observed rightward asymmetry in the cerebellum is in agreement with previous studies as well $(26,27,38)$. Although, a leftward asymmetry has been also reported (10). Regarding the effects of gender on brain asymmetries, while, no region survives Bonferroni correction in post hoc analysis, a few points about observed nominally significant asymmetries (Table.6) worth to be discussed. First, about $12 \%$ of cortical regions show a nominal gender effect on at least one of the metrics. Second, most of exaggerated tendencies to either side belong to male brain. This suggests male probably has a more lateralized brain which is in agreement with previously reported structural as well as functional studies $(1,10,27,39,40)$. Third, the observed asymmetries are relatively subtle, with a Cohen's- $d$ range of -0.19 to 0.19 (Table.6) which is also in line with previous reports $(27,41)$. Fourth, an important part of cortical language as well as visuospatial regions display following gender specific tendencies in male. Transverse temporal cortex, superior temporal and supramarginal gyri as well as inferior parietal cortex present an exaggerated rightward asymmetry in thickness. In addition, surface area displays an exaggerated leftward asymmetry in temporal pole, superior temporal and supramarginal gyri. Finally, an exaggerated leftward asymmetry in gray matter volume of temporal pole and supramarginal gyrus as well as a rightward asymmetry in superior parietal cortex was noted. The observed accentuated asymmetries in male are in agreement with reports indicating more left lateralized language (39) and right lateralized visuospatial functions in males (42). Furthermore, medial orbitofrontal cortex gray matter volume present an opposite tendency in asymmetry between genders i.e. rightward in female and leftward in male $(P<002$, uncorrected). In addition, amygdala present an exaggerated rightward asymmetry in female. Although, several lines of 
evidence suggest that medial prefrontal cortex and amygdala show a differential asymmetrical organization between genders which contribute to distinct cognitive and affective information processing, underlying mechanisms are far from being clear (43-46). The presence $(10,13,14,47)$ or absence of a significant effect of gender on asymmetry $(18,27,28,31,34,48)$ has been frequently reported. In addition of previous mentioned reasons of inconsistencies, this could reflect the fact that asymmetry as a principal developmental feature of human brain follows similar trajectories in both genders yielding only to subtle differences $(18,41)$. In addition, some reports suggest that gender effects on asymmetry might change with age (17). Furthermore, since the absolute values of intracranial volume as well as body size are larger in males, it is challenging to differentiate gender effects on asymmetry from those related to above mentioned factors $(13,31,49)$. Finally, we did not observed a significant age effects on the asymmetry indices of structures studied. However, this obviously would not preclude a relationship between age and asymmetry as participants in this project has been chosen from young adults. This study, for the first time, has provided the asymmetry picture of whole brain structures in unprecedented number of carefully chosen young adults yielding to highly significant results. This provides a basis which would help to understand better this important hallmark of human brain.

\section{Acknowledgements}

The project was supported by funds from Shahid Behehsti University. Data were provided by the Human Connectome Project, WU-Minn Consortium (Principal Investigators: David Van Essen and Kamil Ugurbil; 1U54MH091657) funded by the $16 \mathrm{NIH}$ Institutes and 
bioRxiv preprint doi: https://doi.org/10.1101/2020.12 03.409805; this version posted December 4, 2020. The copyright holder for this preprint

(which was not certified by peer review) is the author/funder, who has granted bioRxiv a license to display the preprint in perpetuity. It is made available under aCC-BY-NC-ND 4.0 International license.

Centers that support the NIH Blueprint for Neuroscience Research; and by the McDonnell

Center for Systems Neuroscience at Washington University. 


\section{References}

1. A. W. Toga, P. M. Thompson, Mapping brain asymmetry. Nat Rev Neurosci 4, 37-48 (2003).

2. $\quad$ P. M. Thompson et al., Cortical variability and asymmetry in normal aging and Alzheimer's disease. Cereb Cortex 8, $492-509$ (1998).

3. B. O. Turner, N. Marinsek, E. Ryhal, M. B. Miller, Hemispheric lateralization in reasoning. Ann N Y Acad Sci 1359, 47-64 (2015).

4. I. Altarelli et al., Planum temporale asymmetry in developmental dyslexia: Revisiting an old question. Hum Brain Mapp 35, 5717-5735 (2014).

5. R. M. Willems, M. V. Peelen, P. Hagoort, Cerebral lateralization of face-selective and body-selective visual areas depends on handedness. Cereb Cortex 20, 1719-1725 (2010).

6. T. J. Crow, Temporal lobe asymmetries as the key to the etiology of schizophrenia. Schizophr Bull 16, 433-443 (1990).

7. K. Yucel et al., Increased subgenual prefrontal cortex size in remitted patients with major depressive disorder. Psychiatry Res 173, 71-76 (2009).

8. K. Amunts, L. Jäncke, H. Mohlberg, H. Steinmetz, K. Zilles, Interhemispheric asymmetry of the human motor cortex related to handedness and gender. Neuropsychologia 38, 304$312(2000)$.

9. E. Goldberg et al., Hemispheric asymmetries of cortical volume in the human brain. Cortex 49, 200-210 (2013).

10. C. D. Good et al., Cerebral asymmetry and the effects of sex and handedness on brain structure: a voxel-based morphometric analysis of 465 normal adult human brains. Neuroimage 14, 685-700 (2001).

11. T. Guadalupe et al., Human subcortical brain asymmetries in 15,847 people worldwide reveal effects of age and sex. Brain Imaging Behav 11, 1497-1514 (2017).

12. X. Kang, T. J. Herron, A. D. Cate, E. W. Yund, D. L. Woods, Hemispherically-unified surface maps of human cerebral cortex: reliability and hemispheric asymmetries. PLoS One 7, e45582 (2012).

13. X. Z. Kong et al., Mapping cortical brain asymmetry in 17,141 healthy individuals worldwide via the ENIGMA Consortium. Proc Natl Acad Sci U S A 115, E5154-e5163 (2018).

14. K. J. Plessen, K. Hugdahl, R. Bansal, X. Hao, B. S. Peterson, Sex, age, and cognitive correlates of asymmetries in thickness of the cortical mantle across the life span. $J$ Neurosci 34, 6294-6302 (2014).

15. M. W. Chee, H. Zheng, J. O. Goh, D. Park, B. P. Sutton, Brain structure in young and old East Asians and Westerners: comparisons of structural volume and cortical thickness. $J$ Cogn Neurosci 23, 1065-1079 (2011).

16. V. A. Kovalev, F. Kruggel, D. Y. von Cramon, Gender and age effects in structural brain asymmetry as measured by MRI texture analysis. Neuroimage 19, 895-905 (2003).

17. P. Shaw et al., Development of cortical asymmetry in typically developing children and its disruption in attention-deficit/hyperactivity disorder. Arch Gen Psychiatry 66, 888-896 (2009).

18. D. Zhou, C. Lebel, A. Evans, C. Beaulieu, Cortical thickness asymmetry from childhood to older adulthood. Neuroimage 83, 66-74 (2013).

19. D. C. Van Essen et al., The WU-Minn Human Connectome Project: an overview. Neuroimage 80, 62-79 (2013).

20. K. C. Bickart, C. I. Wright, R. J. Dautoff, B. C. Dickerson, L. F. Barrett, Amygdala volume and social network size in humans. Nat Neurosci 14, 163-164 (2011). 
21. E. Koechlin, A. Danek, Y. Burnod, J. Grafman, Medial prefrontal and subcortical mechanisms underlying the acquisition of motor and cognitive action sequences in humans. Neuron 35, 371-381 (2002).

22. A. A. Utter, M. A. Basso, The basal ganglia: an overview of circuits and function. Neurosci Biobehav Rev 32, 333-342 (2008).

23. M. F. Glasser et al., The Human Connectome Project's neuroimaging approach. Nat Neurosci 19, 1175-1187 (2016).

24. C. Tanaka, M. Matsui, A. Uematsu, K. Noguchi, T. Miyawaki, Developmental trajectories of the fronto-temporal lobes from infancy to early adulthood in healthy individuals. Dev Neurosci 34, 477-487 (2012).

25. S. Maingault, N. Tzourio-Mazoyer, B. Mazoyer, F. Crivello, Regional correlations between cortical thickness and surface area asymmetries: A surface-based morphometry study of 250 adults. Neuropsychologia 93, 350-364 (2016).

26. P. Y. Hervé, F. Crivello, G. Perchey, B. Mazoyer, N. Tzourio-Mazoyer, Handedness and cerebral anatomical asymmetries in young adult males. Neuroimage 29, 1066-1079 (2006).

27. X. Kang, T. J. Herron, M. Ettlinger, D. L. Woods, Hemispheric asymmetries in cortical and subcortical anatomy. Laterality 20, 658-684 (2015).

28. E. Luders et al., Hemispheric asymmetries in cortical thickness. Cereb Cortex 16, 12321238 (2006).

29. C. Chiarello, D. Vazquez, A. Felton, A. McDowell, Structural asymmetry of the human cerebral cortex: Regional and between-subject variability of surface area, cortical thickness, and local gyrification. Neuropsychologia 93, 365-379 (2016).

30. K. Koelkebeck et al., The contribution of cortical thickness and surface area to gray matter asymmetries in the healthy human brain. Hum Brain Mapp 35, 6011-6022 (2014).

31. O. C. Lyttelton et al., Positional and surface area asymmetry of the human cerebral cortex. Neuroimage 46, 895-903 (2009).

32. M. S. Panizzon et al., Distinct genetic influences on cortical surface area and cortical thickness. Cereb Cortex 19, 2728-2735 (2009).

33. M. Meyer, F. Liem, S. Hirsiger, L. Jäncke, J. Hänggi, Cortical surface area and cortical thickness demonstrate differential structural asymmetry in auditory-related areas of the human cortex. Cereb Cortex 24, 2541-2552 (2014).

34. K. E. Watkins et al., Structural asymmetries in the human brain: a voxel-based statistical analysis of 142 MRI scans. Cereb Cortex 11, 868-877 (2001).

35. P. Rakic, Radial unit hypothesis of neocortical expansion. Novartis Found Symp 228, 3042; discussion 42-52 (2000).

36. O. Pedraza, D. Bowers, R. Gilmore, Asymmetry of the hippocampus and amygdala in MRI volumetric measurements of normal adults. J Int Neuropsychol Soc 10, 664-678 (2004).

37. Y. Wang, Q. Xu, J. Luo, M. Hu, C. Zuo, Effects of Age and Sex on Subcortical Volumes. Front Aging Neurosci 11, 259 (2019).

38. C. A. Szabó et al., MR imaging volumetry of subcortical structures and cerebellar hemispheres in temporal lobe epilepsy. AJNR Am J Neuroradiol 27, 2155-2160 (2006).

39. B. A. Shaywitz et al., Sex differences in the functional organization of the brain for language. Nature 373, 607-609 (1995).

40. K. I. Bolla, D. A. Eldreth, J. A. Matochik, J. L. Cadet, Sex-related differences in a gambling task and its neurological correlates. Cereb Cortex 14, 1226-1232 (2004).

41. A. M. Clements et al., Sex differences in cerebral laterality of language and visuospatial processing. Brain Lang 98, 150-158 (2006).

42. V. J. Bourne, Lateralised processing of positive facial emotion: sex differences in strength of hemispheric dominance. Neuropsychologia 43, 953-956 (2005). 
43. C. Zhang, C. C. Dougherty, S. A. Baum, T. White, A. M. Michael, Functional connectivity predicts gender: Evidence for gender differences in resting brain connectivity. Hum Brain Mapp 39, 1765-1776 (2018).

44. J. Reber, D. Tranel, Sex differences in the functional lateralization of emotion and decision making in the human brain. J Neurosci Res 95, 270-278 (2017).

45. D. Tranel, A. Bechara, Sex-related functional asymmetry of the amygdala: preliminary evidence using a case-matched lesion approach. Neurocase 15, 217-234 (2009).

46. L. Cahill et al., Sex-related difference in amygdala activity during emotionally influenced memory storage. Neurobiol Learn Mem 75, 1-9 (2001).

47. T. Guadalupe et al., Asymmetry within and around the human planum temporale is sexually dimorphic and influenced by genes involved in steroid hormone receptor activity. Cortex 62, 41-55 (2015).

48. L. S. Hamilton et al., Asymmetries of cortical thickness: effects of handedness, sex, and schizophrenia. Neuroreport 18, 1427-1431 (2007).

49. E. Bullmore, M. Brammer, I. Harvey, M. Ron, Against the laterality index as a measure of cerebral asymmetry. Psychiatry Res 61, 121-124 (1995). 
Table 1. Global asymmetry index for each metric of cerebral cortex and white matter.

\begin{tabular}{|c|c|c|c|c|c|c|}
\hline & & & \multicolumn{3}{|c|}{$\mathrm{Al}$} & \multirow{2}{*}{$P$} \\
\hline & & & Mean & SD & $\bar{d}$ & \\
\hline \multirow{4}{*}{ Cerebrum } & \multirow{3}{*}{ Cortex } & Thickness & -0.014 & 0.021 & -0.65 & 0.000000 \\
\hline & & Surface Area & -0.006 & 0.011 & -0.54 & 0.000000 \\
\hline & & Gray Volume & -0.018 & 0.019 & -0.98 & 0.000000 \\
\hline & \multicolumn{2}{|l|}{ White Matter } & -0.014 & 0.009 & -1.51 & 0.000000 \\
\hline
\end{tabular}


Cerebrum

\begin{tabular}{l|l} 
& Thickness \\
Cortex & Surface Area \\
Gray Volume
\end{tabular}

White Matter

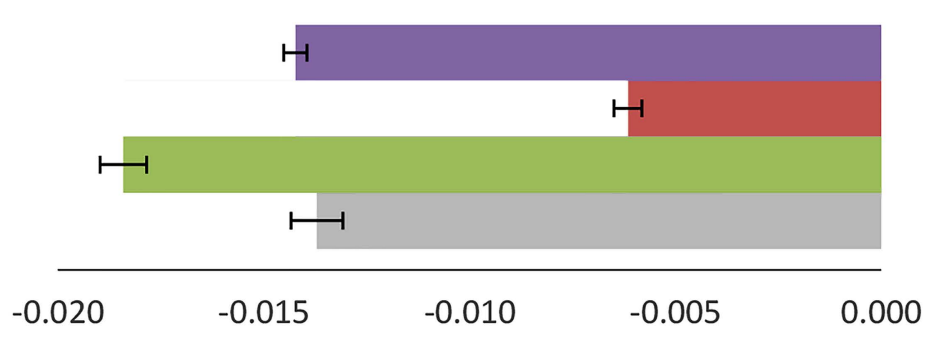

Fig. 1. Global asymmetry indices for metrics of cerebral cortex and white matter volume. Negative asymmetry $(R>L)$ indicates rightward asymmetry. Error bars represent SEM 
Table 2. Asymmetry Index for thickness of each cortical region

\begin{tabular}{|c|c|c|c|c|}
\hline \multirow{2}{*}{ Region } & \multicolumn{3}{|c|}{$\mathrm{Al}$} & \multirow{2}{*}{$P$} \\
\hline & Mean & SD & $d$ & \\
\hline Caudal anterior cingulate cortex & 0.07 & 0.11 & 0.62 & 0.000000 \\
\hline Insula & 0.01 & 0.05 & 0.28 & 0.000000 \\
\hline Posterior cingulate gyrus & 0.01 & 0.07 & 0.21 & 0.000000 \\
\hline Parahippocampal gyrus & 0.01 & 0.08 & 0.08 & 0.009564 \\
\hline Rostral anterior cingulate cortex & 0.00 & 0.07 & 0.06 & 0.042102 \\
\hline Precentral gyrus & 0.00 & 0.03 & 0.04 & 0.204130 \\
\hline Pericalcarine coretx & 0.00 & 0.05 & -0.03 & 0.303877 \\
\hline Cuneus cortex & 0.00 & 0.05 & -0.07 & 0.019816 \\
\hline Frontal pole & -0.01 & 0.07 & -0.12 & 0.000041 \\
\hline Lingual gyrus & -0.01 & 0.05 & -0.16 & 0.000000 \\
\hline Isthmus of cingulate gyrus & -0.03 & 0.17 & -0.16 & 0.000000 \\
\hline Lateral orbitofrontal cortex & -0.01 & 0.04 & -0.18 & 0.000000 \\
\hline Fusiform gyrus & -0.01 & 0.03 & -0.19 & 0.000000 \\
\hline Caudal middle frontal gyrus & -0.01 & 0.03 & -0.21 & 0.000000 \\
\hline Rostral middle frontal gyrus & -0.01 & 0.03 & -0.22 & 0.000000 \\
\hline Paracentral sulcus & -0.01 & 0.04 & -0.27 & 0.000000 \\
\hline Postcentral gyrus & -0.01 & 0.03 & -0.32 & 0.000000 \\
\hline Pars orbitalis & -0.02 & 0.05 & -0.34 & 0.000000 \\
\hline Inferior temporal gyrus & -0.01 & 0.04 & -0.35 & 0.000000 \\
\hline Pars opercularis & -0.02 & 0.04 & -0.40 & 0.000000 \\
\hline Precuneus cortex & -0.01 & 0.03 & -0.42 & 0.000000 \\
\hline Superior frontal gyrus & -0.01 & 0.03 & -0.44 & 0.000000 \\
\hline Entorhinal cortex & -0.04 & 0.08 & -0.47 & 0.000000 \\
\hline Transverse temporal cortex & -0.03 & 0.06 & -0.47 & 0.000000 \\
\hline Superior temporal gyrus & -0.02 & 0.03 & -0.57 & 0.000000 \\
\hline Supramarginal gyrus & -0.02 & 0.03 & -0.58 & 0.000000 \\
\hline Pars triangularis & -0.02 & 0.04 & -0.60 & 0.000000 \\
\hline Lateral occipital coretx & -0.02 & 0.04 & -0.63 & 0.000000 \\
\hline Superior parietal cortex & -0.02 & 0.03 & -0.64 & 0.000000 \\
\hline Middle temporal gyrus & -0.02 & 0.03 & -0.73 & 0.000000 \\
\hline Temporal pole & -0.06 & 0.08 & -0.80 & 0.000000 \\
\hline Banks of superior temporal cortex & -0.04 & 0.05 & -0.82 & 0.000000 \\
\hline Inferior parietal cortex & -0.03 & 0.03 & -0.95 & 0.000000 \\
\hline Medial orbitofrontal cortex & -0.07 & 0.06 & -1.15 & 0.000000 \\
\hline
\end{tabular}


Medial orbitofrontal cortex

Temporal pole

Banks of superior temporal cortex

Entorhinal cortex

Transverse temporal cortex

Inferior parietal cortex

Isthmus of cingulate gyrus

Middle temporal gyrus

Pars triangularis

Lateral occipital coretx

Pars orbitalis

Superior temporal gyrus

Supramarginal gyrus

Superior parietal cortex

Pars opercularis

Precuneus cortex

Inferior temporal gyrus

Paracentral sulcus

Superior frontal gyrus

Postcentral gyrus

Frontal pole

Lingual gyrus

Caudal middle frontal gyrus

Lateral orbitofrontal cortex

Fusiform gyrus

Rostral middle frontal gyrus

Cuneus cortex

Pericalcarine coretx

Precentral gyrus

Rostral anterior cingulate cortex

Parahippocampal gyrus

Posterior cingulate gyrus

Insula

Caudal anterior cingulate cortex

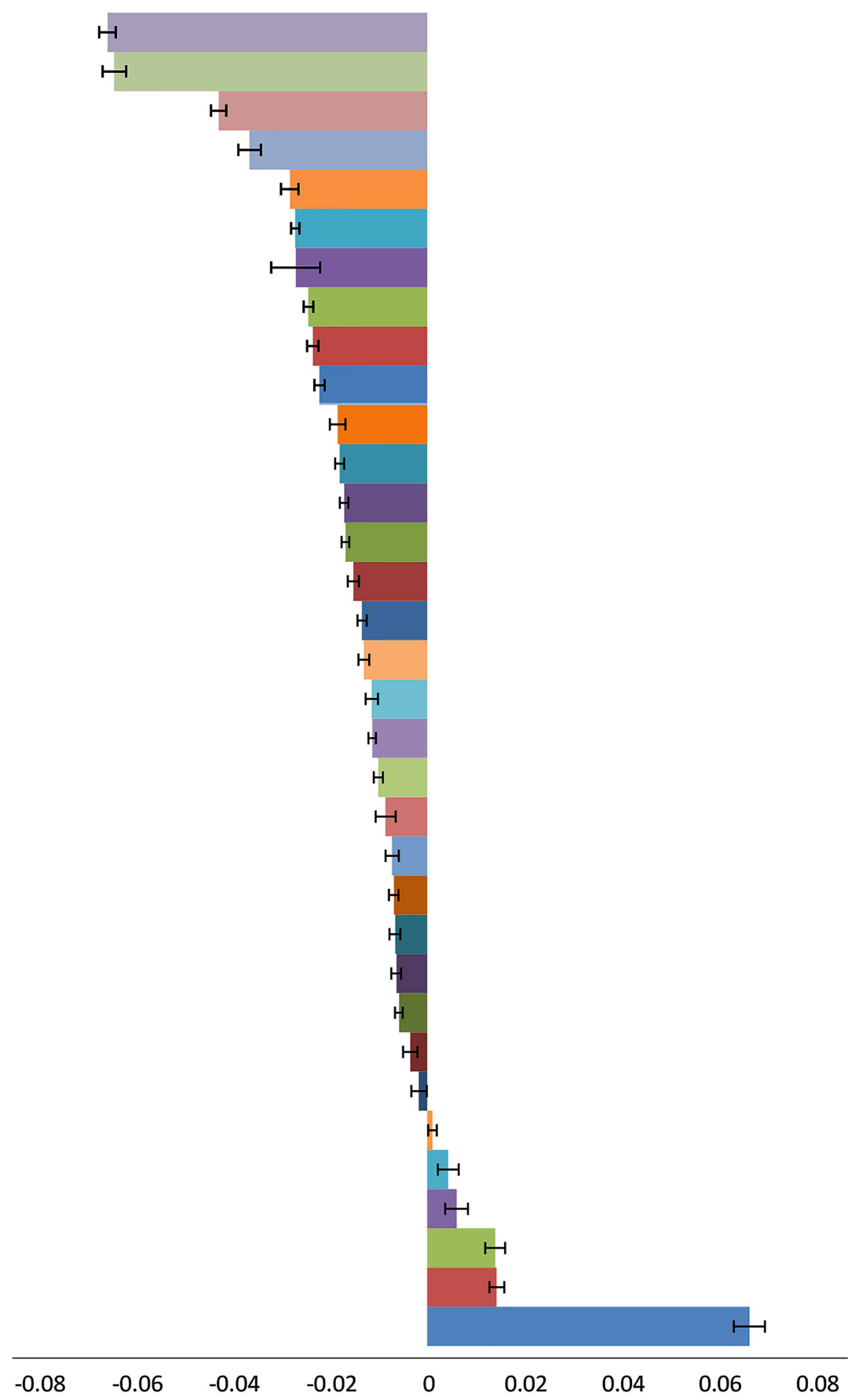

Fig. 2. Asymmetry indices of thickness in cortical regions. Positive asymmetry indicates leftward asymmetry $(L>R)$, while negative asymmetry $(R>L)$ indicates rightward asymmetry. Error bars represent SEM. 
Table 3. Asymmetry Index for surface area of each cortical region

\begin{tabular}{|c|c|c|c|c|}
\hline \multirow{2}{*}{ Region } & \multicolumn{3}{|c|}{$\mathrm{Al}$} & \multirow{2}{*}{$P$} \\
\hline & Mean & SD & $d$ & \\
\hline Transverse temporal cortex & 0.30 & 0.15 & 2.02 & 0.000000 \\
\hline Rostral anterior cingulate cortex & 0.26 & 0.20 & 1.33 & 0.000000 \\
\hline Pars opercularis & 0.17 & 0.16 & 1.10 & 0.000000 \\
\hline Temporal pole & 0.13 & 0.14 & 0.97 & 0.000000 \\
\hline Entorhinal cortex & 0.17 & 0.19 & 0.90 & 0.000000 \\
\hline Superior temporal gyrus & 0.05 & 0.07 & 0.66 & 0.000000 \\
\hline Medial orbitofrontal cortex & 0.07 & 0.12 & 0.55 & 0.000000 \\
\hline Caudal middle frontal gyrus & 0.08 & 0.15 & 0.53 & 0.000000 \\
\hline Supramarginal gyrus & 0.06 & 0.12 & 0.52 & 0.000000 \\
\hline Postcentral gyrus & 0.04 & 0.09 & 0.51 & 0.000000 \\
\hline Banks of superior temporal cortex & 0.07 & 0.15 & 0.47 & 0.000000 \\
\hline Isthmus of cingulate gyrus & 0.10 & 0.23 & 0.43 & 0.000000 \\
\hline Lateral orbitofrontal cortex & 0.03 & 0.06 & 0.41 & 0.000000 \\
\hline Inferior temporal gyrus & 0.04 & 0.11 & 0.39 & 0.000000 \\
\hline Superior frontal gyrus & 0.02 & 0.07 & 0.34 & 0.000000 \\
\hline Parahippocampal gyrus & 0.04 & 0.13 & 0.33 & 0.000000 \\
\hline Fusiform gyrus & 0.03 & 0.10 & 0.30 & 0.000000 \\
\hline Lateral occipital cortex & 0.02 & 0.09 & 0.24 & 0.000000 \\
\hline Superior parietal cortex & 0.00 & 0.08 & -0.02 & 0.606280 \\
\hline Lingual gyrus & -0.01 & 0.09 & -0.07 & 0.017981 \\
\hline Posterior cingulate gyrus & -0.03 & 0.16 & -0.18 & 0.000000 \\
\hline Cuneus cortex & -0.02 & 0.14 & -0.18 & 0.000000 \\
\hline Precentral gyrus & -0.02 & 0.07 & -0.22 & 0.000000 \\
\hline Rostral middle frontal gyrus & -0.04 & 0.07 & -0.51 & 0.000000 \\
\hline Precuneus cortex & -0.05 & 0.08 & -0.67 & 0.000000 \\
\hline Caudal anterior cingulate cortex & -0.17 & 0.26 & -0.67 & 0.000000 \\
\hline Insula & -0.06 & 0.08 & -0.70 & 0.000000 \\
\hline Pericalcarine cortex & -0.09 & 0.12 & -0.78 & 0.000000 \\
\hline Paracentral sulcus & -0.13 & 0.13 & -1.04 & 0.000000 \\
\hline Pars triangularis & -0.15 & 0.14 & -1.08 & 0.000000 \\
\hline Middle temporal gyrus & -0.10 & 0.08 & -1.31 & 0.000000 \\
\hline Frontal pole & -0.32 & 0.18 & -1.78 & 0.000000 \\
\hline Inferior parietal cortex & -0.18 & 0.10 & -1.85 & 0.000000 \\
\hline Pars orbitalis & -0.22 & 0.12 & -1.89 & 0.000000 \\
\hline
\end{tabular}


Frontal pole

Pars orbitalis

Inferior parietal cortex

Caudal anterior cingulate cortex

Pars triangularis

Paracentral sulcus

Middle temporal gyrus

Pericalcarine cortex

Insula

Precuneus cortex

Rostral middle frontal gyrus

Posterior cingulate gyrus

Cuneus cortex

Precentral gyrus

Lingual gyrus

Superior parietal cortex

Superior frontal gyrus

Lateral occipital cortex

Lateral orbitofrontal cortex

Fusiform gyrus

Parahippocampal gyrus

Inferior temporal gyrus

Postcentral gyrus

Superior temporal gyrus

Supramarginal gyrus

Medial orbitofrontal cortex

Banks of superior temporal cortex

Caudal middle frontal gyrus

Isthmus of cingulate gyrus

Temporal pole

Pars opercularis

Entorhinal cortex

Rostral anterior cingulate cortex

Transverse temporal cortex

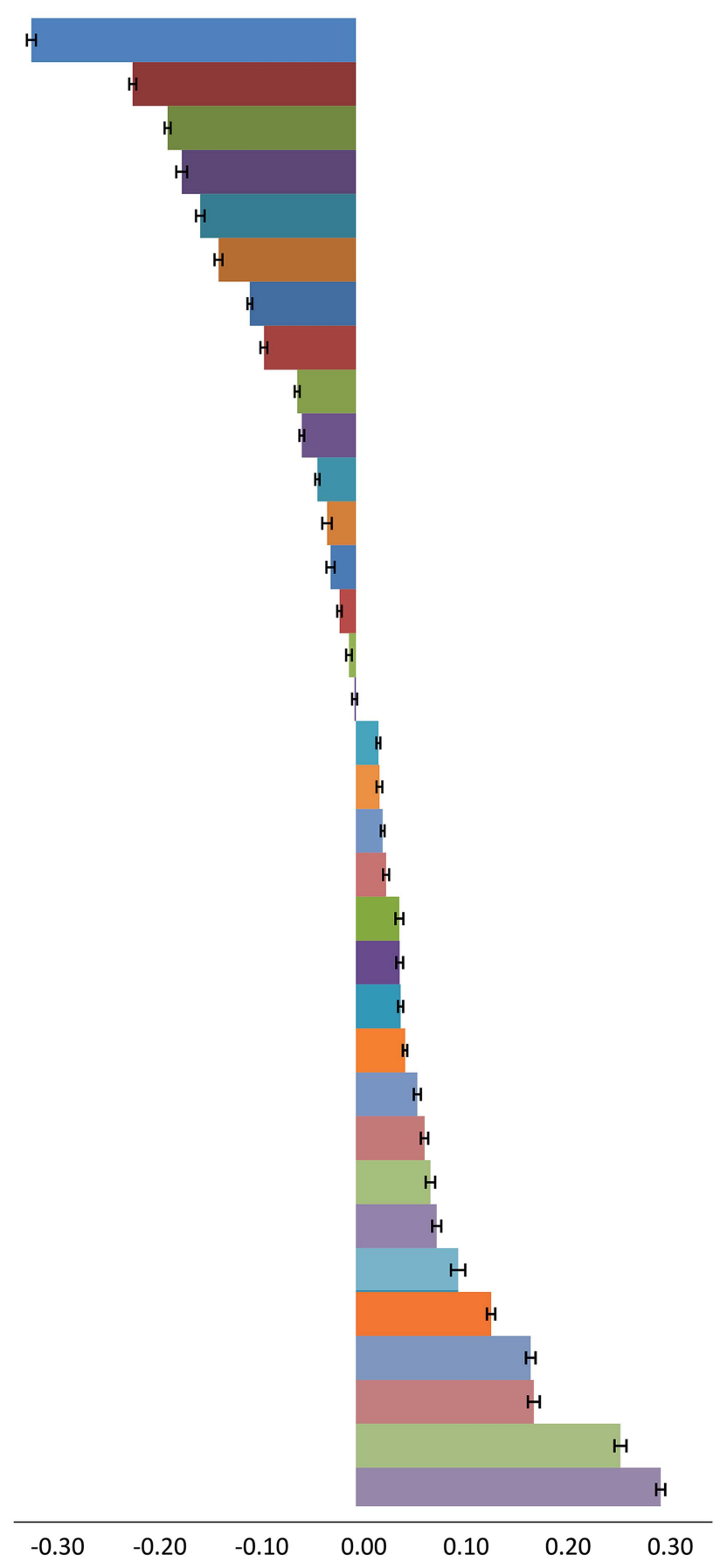

Fig. 3. Asymmetry indices of surface area in cortical regions. Positive asymmetry indicates leftward asymmetry $(L>R)$, while negative asymmetry $(R>L)$ indicates rightward asymmetry. Error bars represent SEM. 
bioRxiv preprint doi: https://doi.org/10.1101/2020.12.03.409805; this version posted December 4, 2020. The copyright holder for this preprint (which was not certified by peer review) is the author/funder, who has granted bioRxiv a license to display the preprint in perpetuity. It is made

Table 4. Asymmetry Index for gray matter volume of each cortical region

Region

Transverse temporal cortex

Rostral anterior cingulate cortex

Pars opercularis

Caudal anterior cingulate cortex

Entorhinal cortex

Parahippocampal gyrus

Supramarginal gyrus

Caudal middle frontal gyrus

Postcentral gyrus

Temporal pole

Isthmus of cingulate gyrus

Superior temporal gyrus

Lateral orbitofrontal cortex

Fusiform gyrus

Inferior temporal gyrus

Superior frontal gyrus

Lateral occipital cortex

Medial orbitofrontal cortex

Posterior cingulate gyrus

Lingual gyrus

Precentral gyrus

Cuneus cortex

Superior parietal cortex

Rostral middle frontal gyrus

Insula

Precuneus cortex

Paracentral sulcus

Pericalcarine cortex

Banks of superior temporal cortex

Pars triangularis

Middle temporal gyrus

Pars orbitalis

Frontal pole

Inferior parietal cortex

$\begin{array}{lll} & \text { Al } & \\ \text { Mean SD } & d\end{array}$

$\begin{array}{llll}0.25 & 0.17 & 1.45 & 0.000000 \\ 0.25 & 0.22 & 1.15 & 0.000000 \\ 0.17 & 0.16 & 1.07 & 0.000000 \\ 0.15 & 0.25 & 0.59 & 0.000000 \\ 0.11 & 0.19 & 0.59 & 0.000000 \\ 0.07 & 0.14 & 0.52 & 0.000000 \\ 0.05 & 0.12 & 0.42 & 0.000000 \\ 0.06 & 0.16 & 0.37 & 0.000000 \\ 0.04 & 0.10 & 0.37 & 0.000000 \\ 0.06 & 0.16 & 0.36 & 0.000000 \\ 0.06 & 0.17 & 0.35 & 0.000000 \\ 0.02 & 0.08 & 0.31 & 0.000000 \\ 0.02 & 0.06 & 0.30 & 0.000000 \\ 0.03 & 0.11 & 0.30 & 0.000000 \\ 0.02 & 0.12 & 0.18 & 0.000000 \\ 0.01 & 0.07 & 0.16 & 0.000000 \\ 0.00 & 0.10 & 0.05 & 0.123153 \\ -0.01 & 0.12 & -0.06 & 0.040444 \\ -0.01 & 0.15 & -0.09 & 0.002520 \\ -0.01 & 0.11 & -0.10 & 0.001182 \\ -0.01 & 0.07 & -0.14 & 0.000007 \\ -0.03 & 0.14 & -0.23 & 0.000000 \\ -0.02 & 0.09 & -0.27 & 0.000000 \\ -0.05 & 0.08 & -0.63 & 0.000000 \\ -0.04 & 0.07 & -0.66 & 0.000000 \\ -0.06 & 0.08 & -0.71 & 0.000000 \\ -0.13 & 0.14 & -0.89 & 0.000000 \\ -0.11 & 0.12 & -0.90 & 0.000000 \\ -0.25 & 0.24 & -1.04 & 0.000000 \\ -0.19 & 0.16 & -1.20 & 0.000000 \\ -0.12 & 0.09 & -1.42 & 0.000000 \\ -0.23 & 0.13 & -1.78 & 0.000000 \\ -0.31 & 0.18 & -1.78 & 0.000000 \\ -0.21 & 0.10 & -2.02 & 0.000000\end{array}$


Frontal pole

Banks of superior temporal cortex

Pars orbitalis

Inferior parietal cortex

Pars triangularis

Paracentral sulcus

Middle temporal gyrus

Pericalcarine cortex

Precuneus cortex

Rostral middle frontal gyrus

Insula

Cuneus cortex

Superior parietal cortex

Posterior cingulate gyrus

Lingual gyrus

Precentral gyrus

Medial orbitofrontal cortex

Lateral occipital cortex

Superior frontal gyrus

Lateral orbitofrontal cortex

Inferior temporal gyrus

Superior temporal gyrus

Fusiform gyrus

Postcentral gyrus

Supramarginal gyrus

Temporal pole

Caudal middle frontal gyrus

Isthmus of cingulate gyrus

Parahippocampal gyrus

Entorhinal cortex

Caudal anterior cingulate cortex

Pars opercularis

Transverse temporal cortex

Rostral anterior cingulate cortex

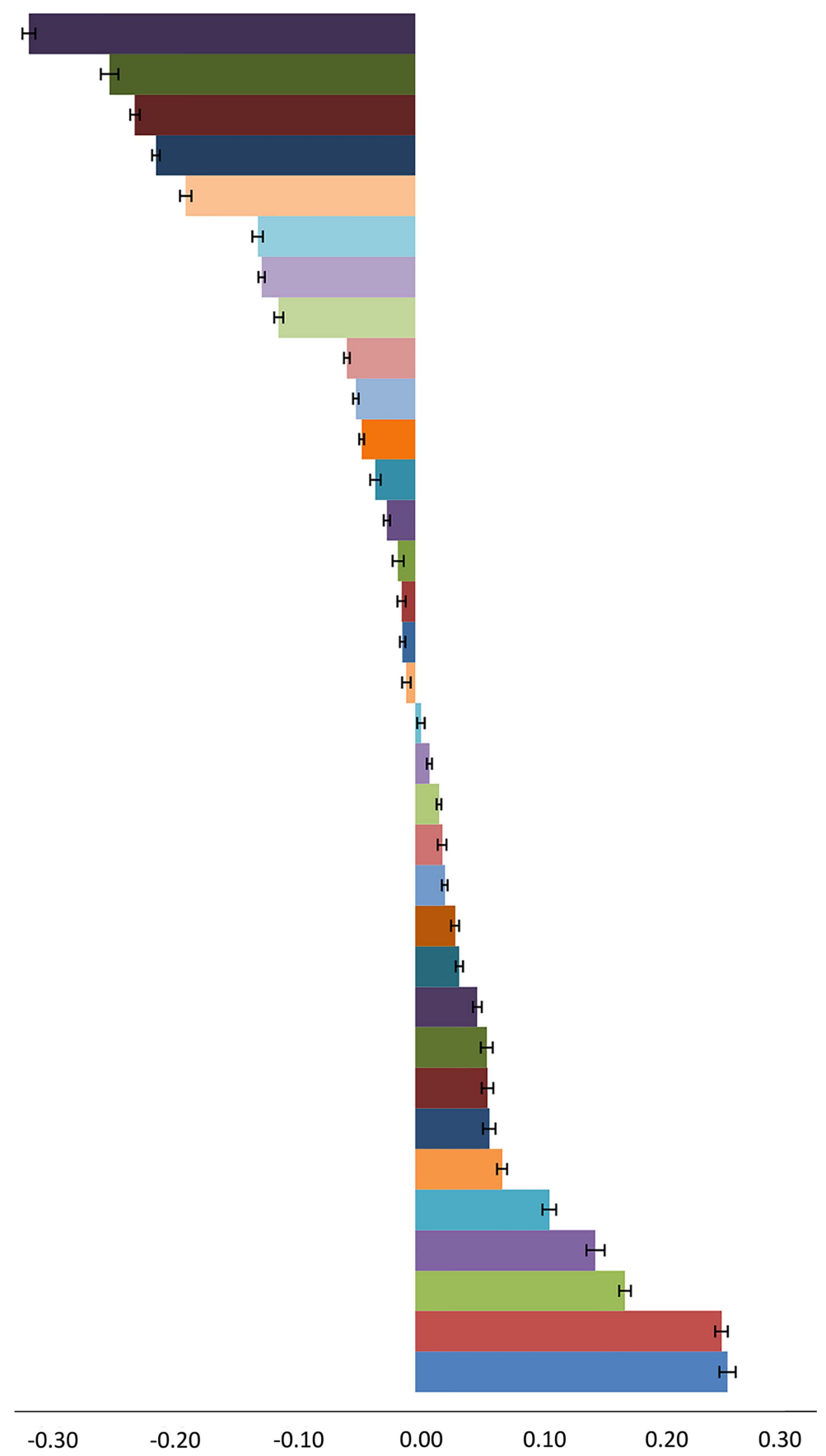

Fig. 4. Asymmetry indices of gray matter volume in cortical regions. Positive asymmetry indicates leftward asymmetry $(L>R)$, while negative asymmetry $(R>L)$ indicates rightward asymmetry. Error bars represent SEM. 
Table 5. Asymmetry Index for hippocampus, cerebellum and subcortical structures

\begin{tabular}{lcccc}
\hline \multirow{1}{*}{ Region } & \multicolumn{3}{c}{ Al } & \multirow{2}{*}{$P$} \\
\cline { 2 - 4 } & Mean & SD & $d$ & \\
\hline Pallidum & -0.10 & 0.13 & -0.79 & 0.000000 \\
Accumbens Area & -0.06 & 0.13 & -0.45 & 0.000000 \\
Amygdala & -0.05 & 0.08 & -0.64 & 0.000000 \\
Caudate Nucleus & -0.03 & 0.04 & -0.75 & 0.000000 \\
Cerebellum Cortex & -0.03 & 0.03 & -0.99 & 0.000000 \\
Cerebellum White Matter & -0.02 & 0.05 & -0.29 & 0.000000 \\
Hippocampus & -0.02 & 0.07 & -0.22 & 0.000000 \\
Putamen & -0.01 & 0.08 & -0.18 & 0.000000 \\
Thalamus & 0.13 & 0.06 & 2.33 & 0.000000 \\
\hline
\end{tabular}




\section{Pallidum}

Accumbens Area

Amygdala

Caudate Nucleus

Cerebellum Cortex

Cerebellum White Matter

Hippocampus

Putamen

Thalamus

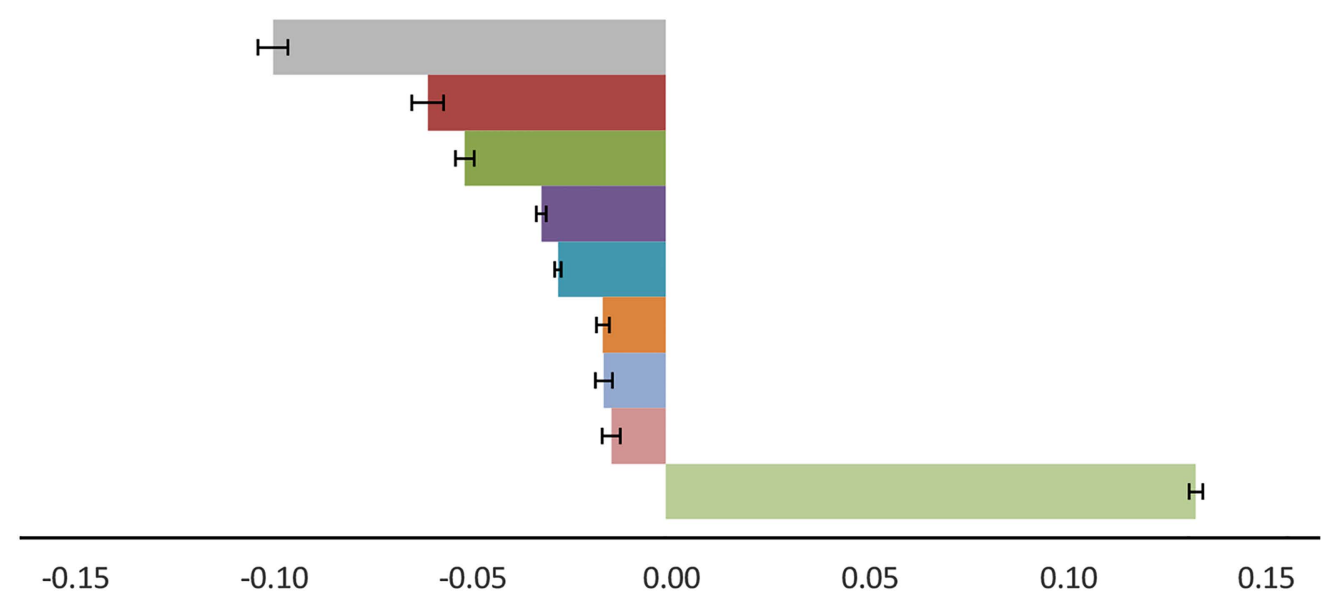

Fig. 5. Asymmetry indices of hippocampus, cerebellum and subcortical regions. Positive asymmetry indicates leftward asymmetry $(L>R)$, while negative asymmetry $(R>L)$ indicates rightward asymmetry. Error bars represent SEM. 


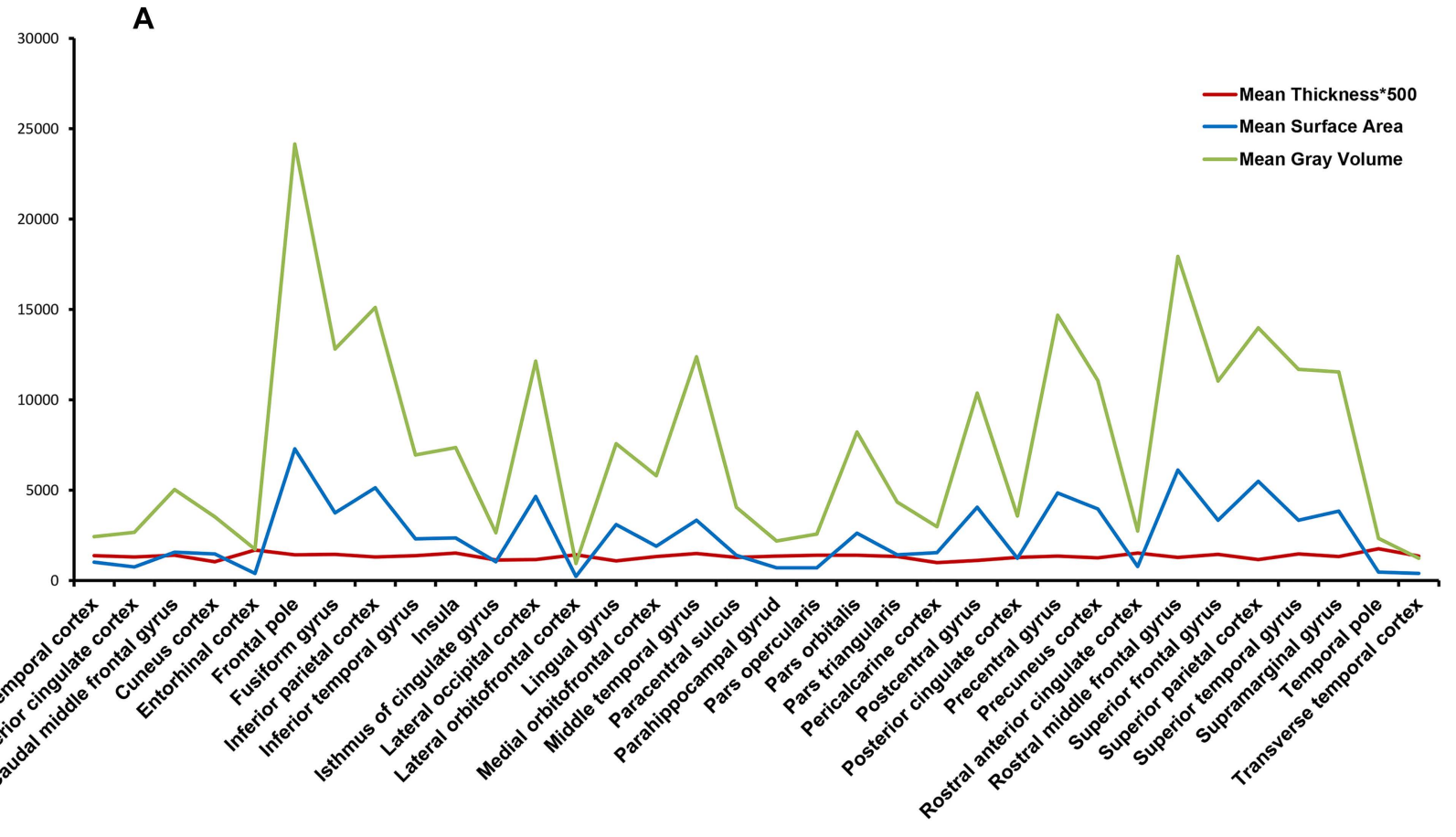

B

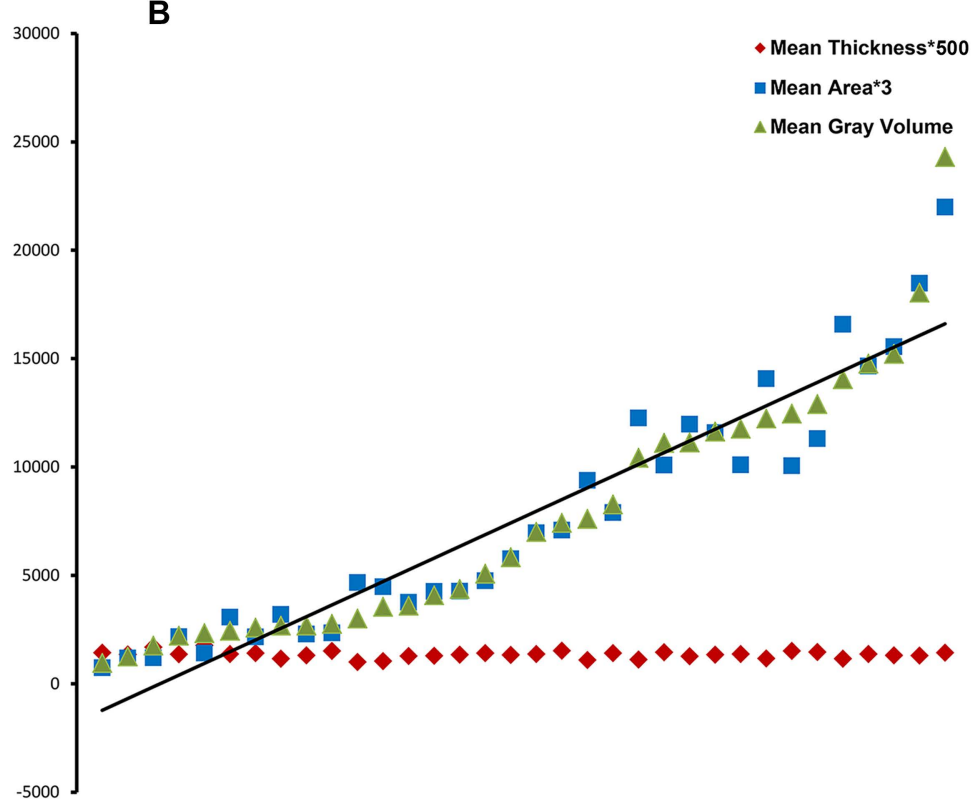

Fig.6. Cortical gray matter volume follows and strongly correlates with surface area across cortical regions $(R 2=0.96, p<0000001)(A, B)$. However, cortical thickness does not show a significant correlation with surface area or gray matter volume (B). In addition, surface area $(\mathrm{CV}=14)$ and gray matter volume $(C V=14)$ show more global variability compared with cortical thickness $(C V=6)(A)$. Thickness and surface area values were multiplied by a constant to make comparison easier. 

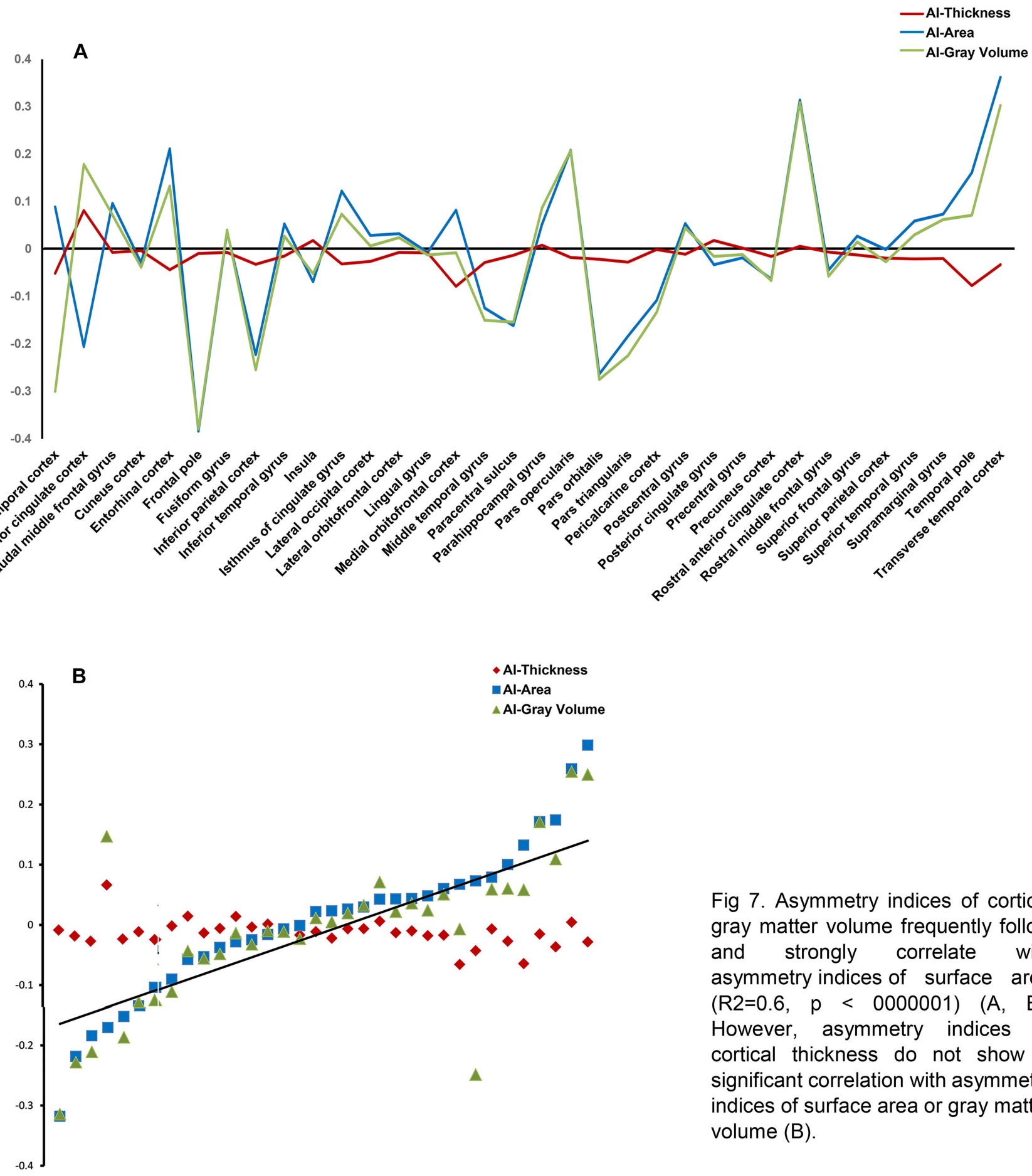

Fig 7. Asymmetry indices of cortical gray matter volume frequently follow and strongly correlate with asymmetry indices of surface area $(R 2=0.6, \quad p<0000001)(A, B)$. However, asymmetry indices of cortical thickness do not show a significant correlation with asymmetry indices of surface area or gray matter volume (B). 
Table 6. Regions with nominally signifiant gender effect on asymmetry index

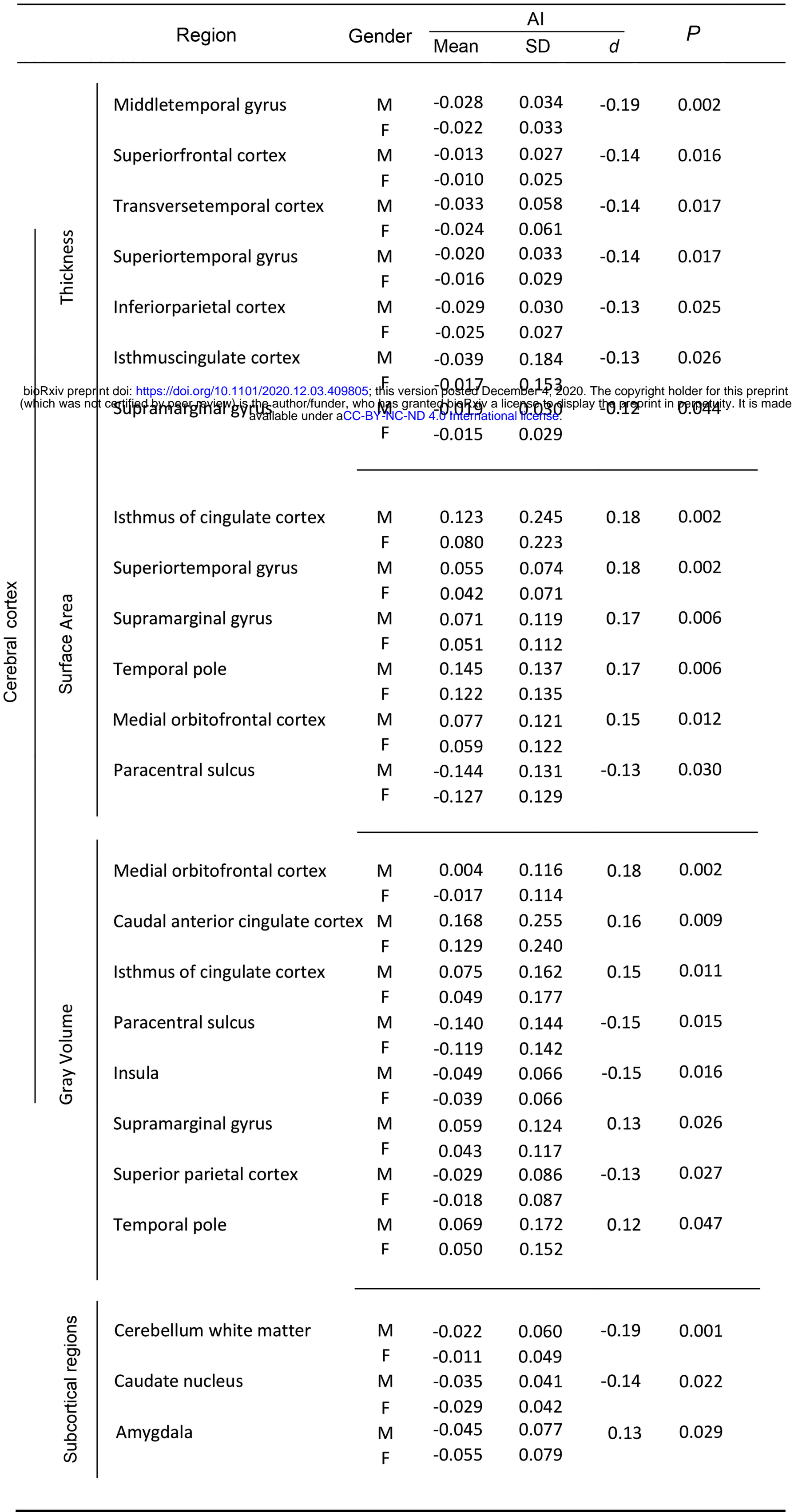

